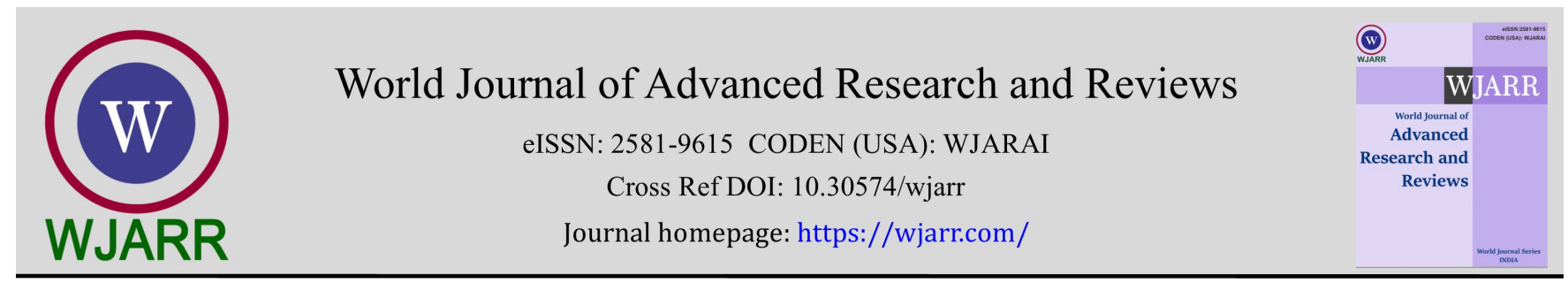

(REVIEW ARTICLE)

Check for updates

\title{
The possible correlation between magnesium deficiency and SARS-CoV-2 infection
}

\author{
Lyly Nazemi 1,2, Mamak Shariat 1,3, Mohammad Mehdi Soltan Dallal 4, 5, Sorourolsadat Daneshfar ${ }^{1}$ and Zahra \\ Farahani $3, *$ \\ ${ }^{1}$ Breastfeeding Research Center, Tehran University of Medical Science, Tehran, Iran. \\ ${ }^{2}$ Department of Clinical Nutrition, School of Nutritional Science and Dietetic, Tehran University of Medical Sciences, Tehran, \\ Iran. \\ ${ }^{3}$ Maternal, Fetal and Neonatal Research Center, Tehran University of Medical Sciences, Tehran, Iran. \\ ${ }^{4}$ Food Microbiology Research Center, Tehran University of Medical Sciences, Tehran, Iran. \\ ${ }^{5}$ Division of Food Microbiology, Department of Pathobiology, School of Public Health, Tehran University of Medical Sciences, \\ Tehran, Iran.
}

World Journal of Advanced Research and Reviews, 2021, 11(01), 073-080

Publication history: Received on 06 June 2021; revised on 10 July 2021; accepted on 12 July 2021

Article DOI: https://doi.org/10.30574/wjarr.2021.11.1.0316

\begin{abstract}
Magnesium (Mg) is an essential electrolyte for living organisms. It is the fourth most abundant mineral in the body. Although Mg is usually found in hard water and high-fiber diets, wide use of soft water and refined flour impose people at risk of Mg deficiency. Imbalances in Mg status and Mg deficiency may result in a variety of complications associated with the immune, cardiovascular, urinary, nervous, and respiratory systems. Because of the important roles of magnesium in the prevention and treatment of many diseases, several studies are focusing on the correlation between Mg status and Coronavirus disease 2019 (COVID-19). The present study reviewed several types of research to indicate the importance of monitoring of $\mathrm{Mg}$ status as well as the possible role of $\mathrm{Mg}$ supplementation for supportive treatment in COVID-19 patients. The results indicated that Mg deficiency could be a risk factor for multi-organ dysfunction in COVID-19 infection. Mg supplementation improves functions of the immune, cardiovascular, nervous, urinary, and respiratory systems through different mechanisms. Suppression of cytokine storm, bronchodilation, antithrombotic function, a decrease of arterial blood pressure, preventing electrolyte imbalance, increase stress resistance are some of the related mechanisms. These findings may provide a reference regarding the possible beneficial effects of the Mg supplement as an inexpensive, safe, and easily available drug for supportive treatment in COVID-19 patients.
\end{abstract}

Keywords: Magnesium; Magnesium Deficiency; COVID-19; Magnesium Supplement

\section{Introduction}

Magnesium (Mg) is the fourth and second most abundant mineral in the body and cell, respectively. Mg plays a crucial role in the structure of the human body and in every essential metabolic reaction. Its concentration in the adult human body is about 25 grams (g). Approximately 50-60\% of all Mg concentration is found in the skeletal system and the remainder is in soft tissues. Less than $1 \%$ of total body $\mathrm{Mg}$ is in blood circulation and only the ionized form of magnesium $\left(\mathrm{Mg}^{2+}\right)$ is physiologically active [1].

Mg is an essential factor for many physiologic mechanisms and pathways like energy production, protein synthesis, ion transport, as well as cell signaling. It has a key role in hundreds of enzymes by its cofactor activity. Mg also affects Vitamin D and Calcium homeostasis. Gastrointestinal diseases, inadequate dietary Mg intake, kidney disorders, chronic alcoholism, and old age are the main risk factors for magnesium deficiency [2].

${ }^{*}$ Corresponding author: Zahra Farahani

Maternal, Fetal and Neonatal Research Center, Tehran University of Medical Sciences, Tehran, Iran.

Copyright (c) 2021 Author(s) retain the copyright of this article. This article is published under the terms of the Creative Commons Attribution Liscense 4.0. 
Low serum Mg concentration correlates to increased risks of several complications including cardiovascular disease, osteoporosis, and metabolic disorders. It has been indicated that Mg by improving insulin sensitivity can decrease the risk of type 2 diabetes mellitus. The role of $\mathrm{Mg}$ supplementation in the prevention of complications following a stroke or heart surgery was also illustrated. $\mathrm{Mg}$ compounds $\left(\mathrm{MgSO}_{4}\right)$ are critically administered in obstetric care preventing seizures in pregnant women with eclampsia. The role of magnesium in preventing brain damage in premature infants is also notable. The use of Mg supplementation for the management of other conditions like asthma or pain is currently under investigation [2-4].

Magnesium content is approximately 50\% higher in the high-fiber diet (green leafy vegetables, unrefined grains, legumes, beans, nuts) and is found in hard water. The tolerable upper intake level related to Mg supplement is reported about $350 \mathrm{mg} /$ day. Although $\mathrm{Mg}$ intoxication is rare, excessive intake may result in several adverse effects especially in subjects with impaired kidney functions. Despite rich sources of dietary Mg, today's wide use of soft water and refined flour puts people at the risk of Mg deficiency $[5,6]$.

Recently, global attention has focused on the pandemic coronavirus (COVID-19). The mortality and morbidity rates are dramatically increasing by COVID-19 [7]. Few investigations showed Mg deficiency in COVID-19 patients and have suggested the possible supportive effects of Mg on COVID-19 outcomes. It was also reported that low Mg status may result in mild to critical transition of COVID-19 manifestations. The effectiveness of Mg replacement in a variety of disorders may show the possibility of supportive treatment with Mg supplementation in COVID-19 cases [7-9].

\section{Hypothesis}

So far, very few studies have assessed the relationship between Mg status andCOVID-19 infection. The present study reviewed and summarized the current knowledge on the possible effects of $\mathrm{Mg}$ on the prevention or management of COVID-19 to discuss the challenges. Thus, firstly, the risk factors correlated to Mg deficiency were explained. Then the effects of $\mathrm{Mg} / \mathrm{Mg}$ deficiency on the immune, cardiovascular, urinary tract, respiratory and nervous systems (which are dramatically affected by COVID-19 infection) were reviewed. Finally, we argued the importance of monitoring of Mg status and treatment of Mg deficiency in COVID-19 patients. The results of this study may provide informative data regarding the effectiveness of Mg supplements as preventative or supportive strategies in populations at risk of COVID19. Moreover, future clinical trials may design and introduce potentially beneficial effects of Mg supplement for infected cases with COVID-19.

\section{Risk factors associated with Mg deficiency}

Mg deficiency is not common in healthy subjects with consuming a balanced diet. $\mathrm{Mg}$ is abundant in plant and animal foods as well as in hard water but the widespread use of soft water and refined flour increases the risk of magnesium deficiency [5,6]. Based on Mg intake, the kidneys regulate urinary excretion of Mg. Several conditions increase the risk of $\mathrm{Mg}$ deficiency. Gastrointestinal disorders (prolonged diarrhea, Crohn's disease, malabsorption syndromes, celiac disease, small intestine inflammations, removal bowel surgeries, radiation), renal disorders, endocrine and metabolic disorders (diabetes mellitus, parathyroid gland disorders, primary aldosteronism), phosphate depletion, excessive lactation, poor dietary intake, alcoholism and long-term use of diuretics may lead to Mg depletion. Furthermore, a decrease of intestinal Mg absorption and an increase of urinary Mg excretion are frequently observed by aging process [10-12].

It seems that COVID-19 disease characterized by multi-organ involvement affects dietary Mg intake, gastrointestinal tract, and renal systems leading to more susceptibility for Mg deficiency. It should be also noted that Mg deficiency by weakening the immune system may make individuals more likely to get COVID-19 infection $[13,14]$.

\section{1. $\mathrm{Mg} / \mathrm{Mg}$ deficiency and immune System}

Mg can affect the immune system by suppressing the cytokine storm and coagulation cascade. It also has a protective role against oxidative stress by activating vitamin $\mathrm{D}$. The beneficial effects of Vitamin D for immune system health have been known for decades [15]. Mg also plays a key role in antiviral immunity and low magnesium levels may account for impaired cytotoxicity [16]. It was noted that high serum Mg concentration could enhance antihistaminic and antiinflammatory responses in patients. Moreover, the association between systemic inflammation and Mg deficiency has been delineated. Mg deficiency causes increases in serum levels of TNF $\alpha$ and inflammatory cytokines with a concomitant decline of production and release of anti-inflammatory cytokines [17-22]. Mg deficiency with its stressor effect makes the body more susceptible to physiological stress resulting in activation of the Hypothalamic-Pituitary-Adrenal (HPA) axis and sympathetic nervous system [23]. This activation may also stimulate the production of oxidative stress and 
NFkB leading to cell apoptosis. Mg deficiency by NFkB signaling triggers increases of inflammatory cytokines and reactive oxygen species through the activation of calcium channels $[22,23]$. These inflammatory responses were confirmed by an animal model study. Inducing Mg deficiency in rats could show leukocyte and macrophage activation, synthesis of inflammatory cytokines, and extensive production of free radicals. Increasing the extracellular Mg levels could alleviate these inflammatory effects [23]. It was also shown that a drop of intracellular free magnesium could induce defective expression of the natural killer activating receptor NKG2D in NK and CD8+ T cells. Mg deficiency can also stimulate hepatic fibrosis and steatosis, possibly through its effect on mast cells [16]. Mg deficiency could accumulate collagen in the cardiovascular system following stress or inflammatory stimuli such as the administration of a hepatotoxic agent [24]. It was demonstrated that $\mathrm{Mg}$ deficiency led to increased proliferation of mast cells in the duodenum and the kidney. The researchers noted that XMEN (X-linked immunodeficiency with magnesium defect, EBV infection, and neoplasia) patients carrying MAGT1 (Mg transporter) mutations had lower free intracellular Mg levels and Mg supplementation could recover the cytotoxicity defect in cytotoxic T-lymphocytes and NK cells [16].

\section{2. $\mathrm{Mg} / \mathrm{Mg}$ deficiency and urinary tract system}

Mg deficiency increases the risk of several diseases like diabetes mellitus type II that negatively affect urinary tract function. An investigation illustrated that low $\mathrm{Mg}$ levels could significantly deteriorate renal function in 550 diabetes mellitus type II cases with not-known kidney disease. The association between different concentrations of Mg in Chronic Kidney Disease (CKD) was also evaluated and results showed that hypomagnesaemia was responsible for more adverse outcomes. It was also suggested that $\mathrm{Mg}$ supplementation may have beneficial effects against calcification and mortality in CKD patients [25]. It should be also mentioned that renal diseases like chronic renal failure or nephrolithiasis can alter Mg balance through excess Mg excretion [26].

\section{3. $\mathrm{Mg} / \mathrm{Mg}$ deficiency and respiratory system}

Several investigations noted that high serum Mg concentration could decrease the incidence of exacerbations of chronic obstructive pulmonary disease (COPD). The possible involving mechanisms may relate to the influences of Mg on the relaxation of bronchial smooth muscle, bronchodilation, anticholinergic, antihistaminic, anti-inflammatory, and mucociliary clearance properties. This finding can explain the correlations between hypomagnesemia with increased airway hyperactivity resulting in chronic obstructive pulmonary disease (COPD) or impaired pulmonary function [17$19,21]$. Micke et al. also showed the correlation between low serum Mg concentration and the exacerbations of COPD [27]. Mg can also induce bronchial smooth muscle cell relaxation by inhibiting cytosolic calcium release in the cells [28]. The beneficial effects of Mg on activation of Vitamin D and its role in pulmonary epithelial and immune system health have been also shown $[15,29]$. The beneficial role of magnesium supplements in respiratory dysfunction was also confirmed by a systematic review with 668 patients. It was shown that hospital admission due to asthma attacks was significantly reduced by the administration of magnesium sulfate. The rates of PEFR (peak expiratory flow rate) and absolute $\mathrm{FEV}_{1}$ (the exhaled volume in the first second of a forced expiration) significantly increased in cases with severe acute asthma [30]. A meta-analysis study revealed that intravenous $\mathrm{Mg}$ sulfate could significantly decrease hospitalization days in asthmatic cases when compared to those treated with a placebo. It also indicated the synergistic effects of Mg sulfate in conjunction with inhaled bronchodilators and systemic steroids in patients with moderate to severe acute asthma [31]. Patients who received nebulized Mg sulfate demonstrated improvement in pulmonary function leading to a decrease in hospitalization. Improved lung function was also significantly notable in cases using nebulized magnesium sulfate with a B2-agonist [32]. The beneficial effect of Mg sulfate in conjunction with $\mathrm{B}_{2}$-agonist agents for adult and children's patients with severe asthma attacks was confirmed by another study [33]. The use of Mg sulfate (nebulized or intravenous) was also recommended for severe and life-threatening asthma exacerbation [34, 35].

\section{4. $\mathrm{Mg} / \mathrm{Mg}$ deficiency and cardiovascular system}

$\mathrm{Mg}$ is an essential element for endothelial function. It was reported that Mg deficiency causes endothelial dysfunction with impaired endothelial-dependent vasodilation. Results of a randomized controlled trial showed that oral Mg supplementation could improve flow-mediated dilation as a marker of endothelial function [36]. An in-vitro study indicated that $\mathrm{Mg}$ deficiency could be a risk factor for cardiovascular diseases through its influences on the increase of pro-inflammatory cytokine interleukin -1a and atherogenesis in endothelial cells [37]. The other study suggested that Mg can act as an antithrombotic agent by inhibiting of platelet reactivity and thrombosis formation [4]. The synthesis of nitric oxide (NO) is also stimulated by Mg resulting in the relaxation of vascular smooth muscle cells [7]. Mg may also play a protective role against vascular calcification via several mechanisms like inhibition of apatite crystals formation, preventing calcium from entering the intracellular space, restoring the balance between the expression of calcification promoters, and inhibitors as well as activating CaSR leading to inhibition of vascular smooth muscle cells calcification [25]. 


\section{5. $\mathrm{Mg} / \mathrm{Mg}$ deficiency and nervous system}

Several studies have demonstrated the cerebroprotective, glioprotective, and neuroprotective effects of $\mathrm{Mg}$. The roles of $\mathrm{Mg}$ in nerve transmission, synaptic plasticity, and excitotoxicity were also well defined. It was indicated that neurological trauma and events may lead to a decrease of $\mathrm{Mg}$ concentration through the intracellular shift and increased urinary excretion. There are notable correlations between hypomagnesaemia and various types of neurological complications like depression, stroke, Parkinson's disease, migraine cerebral vasospasm, convulsion, and Alzheimer's disease [38-42]. Divers' neurological manifestations such as muscle tremor, tetany, hyperreflexia, dystaxia, delirium, headache, and convulsion are also observed with severe hypomagnesemia [41]. An investigation reported that Mg supplementation by its role in brain revascularization can improve outcomes related to cerebral ischemia. The possible involving mechanisms may be associated with calcium antagonist and neuroprotective effects of Mg [43]. Another study demonstrated poor outcomes in traumatic brain injury patients with low serum and high cerebrospinal fluid magnesium levels compared to patients with normal magnesium levels [44].It has been also shown that daily $\mathrm{Mg}$ intake for more than 8 weeks could improve depression status in depressed cases [42].

\section{6. $\mathrm{Mg} / \mathrm{Mg}$ deficiency and COVID-19 infection}

Multiple organs are affected by COVID-19 but the involvement of respiratory, cardiovascular, nervous, immune systems, and kidneys are responsible for the main manifestations and outcome [45]. It is supposed that Mg deficiency could be a risk factor for COVID-19 infection like other complications (cardiovascular disease, diabetes, chronic respiratory disease, old age, and obesity [27]. Hypomagnesemia was reported in $32 \%$ of 134 in- and out-patient cases with COVID19 without underlying disease [46]. An investigation pointed to Mg deficiency as a risk factor for a severe COVID-19. It was indicated that people with low energy reserves due to Mg deficiency were prone to develop severe COVID-19 symptoms. The authors suggested the role of monitoring of Mg status and supplementation in deficient cases for preventing COVID-19 and its complications [9]. It was demonstrated that regular monitoring of ionized magnesium status with subsequent repletion of Mg may be an effective strategy preventing disease progression [13].

Previous investigations suggested that Mg supplementation may help COVID-19 patients by its effects on different systems. Regarding its influences on the immune system, the possible immunomodulatory and anti-inflammatory effects of Mg compounds (Mg sulfate) on the cytokine phase of COVID-19 have been demonstrated [47]. Mg with "calcium-channel blocking" effect may lead to downstream suppression of nuclear factor-K $\beta$, interleukin-6, and Creactive protein resulting in suppression of life-threatening events like cytokine storm in COVID-19 disease. Furthermore, Mg by its ability to activate vitamin D may improve immune system health and protect the body against COVID-19 infection $[13,15]$.

COVID-19 patients severely suffer from respiratory symptoms. The impacts of Mg supplements on alleviation of respiratory complications in COVID-19 subjects have been reported by Sahin et al. The authors demonstrated that the involved mechanism may be relating to the shift of M1 to M2 type macrophages and stimulation of anti-inflammatory cytokine secretion in the respiratory tract [48]. Another study showed that intravenous administration of Mg could suppress severe inflammatory responses in the respiratory tract and prevent worsening the disease courses in COVID19 patients [27]. $\mathrm{Mg}$ as a calcium antagonist can inhibit bronchospasm and results in bronchodilation. $\mathrm{Mg}$ by suppression of inflammatory response and oxidative stress can also alleviate pulmonary symptoms in the COVID-19 patients [7]. The other mechanisms may be associated with mucociliary clearance effects of $\mathrm{Mg}$ that can decrease resistance in the airways and improve lung function in COVID-19 patients. The beneficial pharmacological effects of intravenous Mg administration have been demonstrated on lung function [27]. It was suggested that serum Mg level should be monitored and supplementation of Mg should be initiated in deficient cases during the early phase of COVID19. As Mg may diminish the secondary co-morbidities, prophylaxis with Mg may also be beneficial in populations at risk [9].

It was revealed that COVID-19 like other viral infections influences the endothelial cells and plays a key role in the pathogenesis of cardiovascular dysfunction and associated consequences [49-51]. Mg deficiency may also cause frequent occurrences of thrombotic embolism and coagulation cascade in COVID-19 patients. Mg administration may act as an antithrombotic agent (by suppression of platelet reactivity) to prevent or improve cardiovascular manifestations $[4,52,53]$. These findings show the importance of stabilizing the endothelial status particularly in highrisk patients like COVID-19 patients with pre-existing endothelial dysfunction [48]. The effectiveness of Mg on a variety of cardiovascular complications associated with COVID-19 disease has been also reported by the previous investigation. Decrease of systolic, diastolic, and mean arterial blood pressure, an increase of endothelium-derived NO levels, lowering the risk of ventricular dysfunction, and atrial fibrillation have been shown by Mg supplementation in COVID-19 patients [7]. 
Previous studies have shown that patients with chronic kidney disease were at higher risk of severe COVID-19 disease. A wide range of kidney complications from proteinuria or haematuria to renal failure and the need for haemodialysis has been also indicated among hospitalized COVID-19 patients [54-56]. As all these urinary tract complications are frequently observed among Mg deficient patients, thus it seems Mg supplementation can prevent COVID-19 patients from further deterioration of their condition [7]. Moreover, Covid-19 infection may also exacerbate the hypomagnesaemia through an excess increase of stress hormones and urinary Mg excretion. Preventing or treatment of such an electrolyte imbalance may provide optimal supportive therapy for Covid-19 patients [45, 57].

Regarding the influence of Coronavirus on the nervous system, it should be noted that Coronavirus is detected in the brain tissue as well as in cerebrospinal fluid that can severely impact the nervous system through diverse pathways [58]. COVID-19 patients complain of a wide range of mild to severe neurological manifestations like headache, dizziness, hypogeusia, myalgia, hyposmia, polyneuropathy, myositis, complications related to cerebrovascular events or intracranial infections [59,60]. Moreover, COVID-19 patients severely experience acute and chronic emotional tensions which result in an increase of stress hormones catecholamines and corticosteroids [46, 61,62]. As mentioned before, all these events may lead to the loss of more and more Mg. Besides that, Mg deficiency is one of the main factors for decrease of stress and immune resistance as well as adaptive body reserves. So, it seems that using Mg supplements to correct Mg level in COVID-19 patients can beneficially increase stress resistance and prevent neurological diseases [61]. It was also indicated that $\mathrm{Mg}$ by antagonizing calcium can interfere with calcium functions either at the level of calcium channels, cytosolic influx, calcium-dependent enzymatic activities, inflammatory and blood clotting processes. These properties of Mg may significantly prevent neurological and cerebrovascular pathologies in COVID-19 patients [63].

In brief, there are no pharmacological strategies for the treatment of COVID-19; however, in the present study, we have shown the importance of nutritional strategies. Previous research has demonstrated that magnesium has a wide-range of systemic effects on the body. On the other hand, Mg deficiency has been shown as one of the pathogenic factors for decreasing stress resistance and body's adaptive reserves, thrombotic events, bronchospasm, ETC. So, it is supposed that Mg supplementation may be beneficial in vulnerable populations such as COVID-19 patients. However, relevant research questions such as the prevalence rate of Mg deficiency in COVID-19 patients, correlation between Mg deficiency with the severity of disease or the occurrence of co-morbidities, and the therapeutic doses need to be discussed and determined by future studies and clinical trials.

\section{Conclusion}

In the present study, previous studies were reviewed and the results indicate the positive correlations between $\mathrm{Mg}$ deficiency and multi-organ dysfunctions in COVID-19 disease. The effectiveness of Mg supplementation on improved functions of immune, cardiovascular, urinary tract, respiratory, and nervous systems may provide a reference regarding the possible beneficial effects of Mg for supportive therapy in COVID-19 cases. However, future studies should determine the prevalence rate of Mg deficiency in COVID-19 patients, the therapeutic doses, and the correlation between $\mathrm{Mg}$ supplementation and the severity, co-morbidity, and outcome of the disease.

\section{Compliance with ethical standards}

\section{Acknowledgments}

Dr. L. N and Dr. M.S carried out the design and coordinated the study, participated in most of the experiments. Dr. S.D, Dr. Z.F. and Dr. M.M.S.D. participated in manuscript preparation. All authors have read and approved the content of the manuscript.

This research has been supported by School of Nutritional Science and Dietetic, Tehran University of Medical Sciences and health service.

Our study was approved by the institutional review board of Tehran University of Medical Sciences and according to Helsinki declaration.

\section{Disclosure of conflict of interest}

The authors declare that there is no conflict of interests. 


\section{References}

[1] Erdman Jr JW, Macdonald IA, Zeisel SH. Present knowledge in nutrition: John Wiley \& Sons. 2012.

[2] Gröber U, Schmidt J, Kisters K. Magnesium in prevention and therapy. Nutrients. 2015 Sep;7(9):8199-226.

[3] Champagne CM. Magnesium in hypertension, cardiovascular disease, metabolic syndrome, and other conditions: a review. Nutrition in Clinical Practice. 2008; 23(2): 142-51.

[4] DiNicolantonio JJ, O'Keefe JH, Wilson W. Subclinical magnesium deficiency: a principal driver of cardiovascular disease and a public health crisis. Open heart. 2018; 5(1): e000668.

[5] Flink EB. Nutritional aspects of magnesium metabolism. Western Journal of Medicine. 1980; 133(4): 304.

[6] McCarty MF. Magnesium may mediate the favorable impact of whole grains on insulin sensitivity by acting as a mild calcium antagonist. Medical hypotheses. 2005; 64(3): 619-27.

[7] Tang C-F, Ding H, Jiao R-Q, Wu X-X, Kong L-D. Possibility of magnesium supplementation for supportive treatment in patients with COVID-19. European Journal of Pharmacology. 2020; 173546.

[8] Iotti S, Wolf F, Mazur A, Maier JA. The COVID-19 pandemic: is there a role for magnesium? Hypotheses and perspectives. Magnesium Research. 2020; 1(1).

[9] Van Kempen TA, Deixler E. SARS-CoV-2: influence of phosphate and magnesium, moderated by vitamin D, on energy (ATP) metabolism and on severity of COVID-19. American Journal of Physiology-Endocrinology and Metabolism. 2021; 320(1): E2-E6.

[10] Intakes IoMSCotSEoDR. Dietary reference intakes. Dietary Reference Intakes for Calcium, Phosphorus, Magnesium, Vitamin D, and Fluoride. 1997.

[11] Ross AC, Caballero B, Cousins RJ, Tucker KL, Ziegler TR. Modern nutrition in health and disease: Lippincott Williams \& Wilkins. 2012.

[12] Moshfegh A, Goldman J, Ahuja J, Rhodes D, LaComb R. What we eat in America, NHANES. 2005-2006: usual nutrient intakes from food and water compared to 1997 dietary reference intakes for vitamin D, calcium, phosphorus, and magnesium. US Department of Agriculture, Agricultural Research Service. 2009.

[13] Wallace TC. Combating COVID-19 and building immune resilience: a potential role for magnesium nutrition? Journal of the American College of Nutrition. 2020; 39(8): 685-93.

[14] Richardson DP, Lovegrove JA. Nutritional status of micronutrients as a possible and modifiable risk factor for COVID-19: a UK perspective. British journal of nutrition. 2021; 125(6): 678-84.

[15] DiNicolantonio JJ, O'Keefe JH, McCarty MF. Supplemental N-acetylcysteine and other measures that boost intracellular glutathione can downregulate interleukin-1 $\beta$ signalling: a potential strategy for preventing cardiovascular events? : Archives of Disease in childhood. 2017.

[16] Chaigne-Delalande B, Li F-Y, O'Connor GM, Lukacs MJ, Jiang P, Zheng L, et al. Mg2+ regulates cytotoxic functions of NK and CD8 T cells in chronic EBV infection through NKG2D. Science. 2013; 341(6142): 186-91.

[17] Bhatt SP, Khandelwal P, Nanda S, Stoltzfus JC, Fioravanti GT. Serum magnesium is an independent predictor of frequent readmissions due to acute exacerbation of chronic obstructive pulmonary disease. Respiratory medicine. 2008; 102(7): 999-1003.

[18] Aziz HS, Blamoun AI, Shubair MK, Ismail MM, DeBari VA, Khan MA. Serum magnesium levels and acute exacerbation of chronic obstructive pulmonary disease: a retrospective study. Annals of Clinical \& Laboratory Science. 2005; 35(4): 423-7.

[19] Gumus A, Haziroglu M, Gunes Y. Association of serum magnesium levels with frequency of acute exacerbations in chronic obstructive pulmonary disease: a prospective study. Pulmonary medicine. 2014; 2014.

[20] Ni H, Naing C, Aye SZ. Magnesium sulfate for acute exacerbations of chronic obstructive pulmonary disease. The Cochrane Database of Systematic Reviews. 2020; 2020(1).

[21] Bhaumik S, Choudhury A, Chakrabarti D, Dey P. Study of serum magnesium level in COPD and its impact on exacerbation of COPD. J Evid Based Med Healthcare. 2019; 6(16): 1235-9.

[22] Romani AM. Magnesium in health and disease. Interrelations between essential metal ions and human diseases. 2013; 49-79. 
[23] Rayssiguier Y, Libako P, Nowacki W, Rock E. Magnesium deficiency and metabolic syndrome: stress and inflammation may reflect calcium activation. Magnesium research. 2010; 23(2): 73-80.

[24] Rayssiguier Y, Chevalier F, Bonnet M, Kopp J, Durlach J. Influence of magnesium deficiency on liver collagen after carbon tetrachloride or ethanol administration to rats. The Journal of nutrition. 1985; 115(12): 1656-62.

[25] De Francisco A, Rodríguez M. Magnesium-its role in CKD. Nefrologia. 2013; 33(3): 389-99.

[26] Musso CG. Magnesium metabolism in health and disease. International urology and nephrology. 2009; 41(2): 357-62.

[27] Micke O, Vormann J, Kisters K. Magnesium deficiency and COVID-19-What are the links? Trace Elements and Electrolytes. 2020; 37(3): 103.

[28] Gourgoulianis K, Chatziparasidis G, Chatziefthimiou A, Molyvdas P-A. Magnesium as a relaxing factor of airway smooth muscles. Journal of aerosol medicine. 2001; 14(3): 301-7.

[29] Pallath MM, Ahirwar AK, Tripathi SC, Asia P, Sakarde A, Gopal N. COVID-19 and nutritional deficiency: A review of existing knowledge. Hormone Molecular Biology and Clinical Investigation. 2021.

[30] Rowe BH, Bretzlaff JA, Bourdon C, Bota GW, Camargo Jr CA. Intravenous magnesium sulfate treatment for acute asthma in the emergency department: a systematic review of the literature. Annals of emergency medicine. 2000; 36(3): 181-90.

[31] Heraghty J, Henderson A. Highlights in asthma 2005. Archives of disease in childhood. 2006; 91(5): 422-5.

[32] Blitz M, Blitz S, Hughes R, Diner B, Beasley R, Knopp J, et al. Aerosolized magnesium sulfate for acute asthma: a systematic review. Chest. 2005; 128(1): 337-44.

[33] Shan Z, Rong Y, Yang W, Wang D, Yao P, Xie J, et al. Intravenous and nebulized magnesium sulfate for treating acute asthma in adults and children: a systematic review and meta-analysis. Respiratory medicine. 2013; 107(3): 321-30.

[34] Song W-J, Kang M-G, Chang Y-S, Cho S-H. Epidemiology of adult asthma in Asia: toward a better understanding. Asia Pacific Allergy. 2014; 4(2): 75.

[35] Sellers W. Inhaled and intravenous treatment in acute severe and life-threatening asthma. British journal of anaesthesia. 2013; 110(2): 183-90.

[36] Mofrad MD, Djafarian K, Mozaffari H, Shab-Bidar S. Effect of magnesium supplementation on endothelial function: A systematic review and meta-analysis of randomized controlled trials. Atherosclerosis. 2018; 273: 98-105.

[37] Ferrè S, Mazur A, Maier JA. Low-magnesium induces senescent features in cultured human endothelial cells. Magnesium research. 2007; 20(1): 66-71.

[38] Saver JL, Starkman S, Eckstein M, Stratton SJ, Pratt FD, Hamilton S, et al. Prehospital use of magnesium sulfate as neuroprotection in acute stroke. New England Journal of Medicine. 2015; 372(6): 528-36.

[39] Polderman KH, van Zanten AR, Girbes AR. The importance of magnesium in critically ill patients: a role in mitigating neurological injury and in the prevention of vasospasms. Intensive care medicine. 2003; 29(7): 12023.

[40] Kirkland AE, Sarlo GL, Holton KF. The Role of Magnesium in Neurological Disorders. Nutrients. 2018; 10(6).

[41] Wenwen XUE, Jing YOU, Yingchao SU, Qinglu W. The Effect of Magnesium Deficiency on Neurological Disorders: A Narrative Review Article. Iranian Journal of Public Health. 2019; 48(3).

[42] Rajizadeh A, Mozaffari-Khosravi H, Yassini-Ardakani M, Dehghani A. Effect of magnesium supplementation on depression status in depressed patients with magnesium deficiency: A randomized, double-blind, placebocontrolled trial. Nutrition. 2017; 35: 56-60.

[43] Xu R, Wang L, Sun L, Dong J. Neuroprotective effect of magnesium supplementation on cerebral ischemic diseases. Life Sciences. 2021; 272: 119257.

[44] Stippler M, Fischer MR, Puccio AM, Wisniewski SR, Carson-Walter EB, Dixon CE, et al. Serum and Cerebrospinal Fluid Magnesium in Severe Traumatic Brain Injury Outcome. Journal of Neurotrauma. 2007; 24(8): $1347-54$.

[45] Jain U. Effect of COVID-19 on the Organs. Cureus. Aug 2020; 12(8). 
[46] Sarvazad H, Cahngaripour S, Roozbahani NE, Izadi B. Evaluation of electrolyte status of sodium, potassium and magnesium, and fasting blood sugar at the initial admission of individuals with COVID-19 without underlying disease in Golestan Hospital, Kermanshah. New Microbes and New Infections. 2020; 38: 100807.

[47] Parichehr P, Guitti P. Magnesium Sulfate: A Potential Adjuvant Treatment on COVID-19. Frontiers in Emergency Medicine. 2020; 5(1).

[48] Sahin E, Orhan C, Uckun FM, Sahin K. Clinical Impact Potential of Supplemental Nutrients as Adjuncts of Therapy in High-Risk COVID-19 for Obese Patients. Frontiers in Nutrition. 2020; 7(205).

[49] Varga Z, Flammer AJ, Steiger P, Haberecker M, Andermatt R, Zinkernagel AS, et al. Endothelial cell infection and endotheliitis in COVID-19. The Lancet. 2020; 395(10234): 1417-8.

[50] Joly BS, Siguret V, Veyradier A. Understanding pathophysiology of hemostasis disorders in critically ill patients with COVID-19. Intensive care medicine. 2020; 46(8): 1603-6.

[51] Ackermann M, Verleden SE, Kuehnel M, Haverich A, Welte T, Laenger F, et al. Pulmonary vascular endothelialitis, thrombosis, and angiogenesis in Covid-19. New England Journal of Medicine. 2020; 383(2): 120-8.

[52] Violi F, Pastori D, Cangemi R, Pignatelli P, Loffredo L. Hypercoagulation and antithrombotic treatment in coronavirus 2019: a new challenge. Thrombosis and haemostasis. 2020; 120(6): 949.

[53] Wichmann D, Sperhake J-P, Lütgehetmann M, Steurer S, Edler C, Heinemann A, et al. Autopsy findings and venous thromboembolism in patients with COVID-19: a prospective cohort study. Annals of internal medicine. 2020; 173(4): 268-77.

[54] D'Marco L, Puchades MJ, Romero-Parra M, Gimenez-Civera E, Soler MJ, Ortiz A, et al. Coronavirus disease 2019 in chronic kidney disease. Clinical kidney journal. 2020; 13(3): 297-306.

[55] Henry BM, Lippi G. Chronic kidney disease is associated with severe coronavirus disease 2019 (COVID-19) infection. International urology and nephrology. 2020; 52(6): 1193-4.

[56] COVID C, Team R, COVID C, Team R, COVID C, Team R, et al. Preliminary estimates of the prevalence of selected underlying health conditions among patients with coronavirus disease 2019-United States, February 12-March 28, 2020. Morbidity and Mortality Weekly Report. 2020; 69(13): 382.

[57] Kraft MD, Btaiche IF, Sacks GS, Kudsk KA. Treatment of electrolyte disorders in adult patients in the intensive care unit. American Journal of Health-System Pharmacy. 2005; 62(16): 1663-82.

[58] Wu Y, Xu X, Chen Z, Duan J, Hashimoto K, Yang L, et al. Nervous system involvement after infection with COVID19 and other coronaviruses. Brain, Behavior, and Immunity. 2020; 87: 18-22.

[59] Tsivgoulis G, Palaiodimou L, Katsanos AH, Caso V, Köhrmann M, Molina C, et al. <? covid19?> Neurological manifestations and implications of COVID-19 pandemic. Therapeutic advances in neurological disorders. 2020; 13: 1756286420932036.

[60] Abboud H, Abboud FZ, Kharbouch H, Arkha Y, El Abbadi N, El Ouahabi A. COVID-19 and SARS-Cov-2 Infection: Pathophysiology and Clinical Effects on the Nervous System. World neurosurgery. 2020.

[61] Vasil'EvYuriy L. Possible prospects for using modern magnesium preparations for increasing stress resistance during COVID-19 pandemic. Research Results in Pharmacology. 2020; 6(4).

[62] Salari N, Hosseinian-Far A, Jalali R, Vaisi-Raygani A, Rasoulpoor S, Mohammadi M, et al. Prevalence of stress, anxiety, depression among the general population during the COVID-19 pandemic: a systematic review and metaanalysis. Globalization and health. 2020; 16(1): 1-11.

[63] Errasfa M. Magnesium therapeutic potential against Covid-19: Could it be an" All-in-one" therapy? Magnesium Research. 2021; 1(1). 